\title{
Local $\mathrm{O}_{\delta}$ probing in the high- $T_{c}$ superconductor $\mathrm{HgBa}_{2} \mathrm{CuO}_{4+\delta}$
}

J. G. Correia* and ISOLDE Collaboration

CERN-EP/SC, CH-1211 Geneva 23, Switzerland

J. P. Araújo

IFIMUP, Departamento de Física, FCP, Rua do Campo Alegre 687, P-4150 Porto, Portugal

S. M. Loureiro

Department of Chemistry, Princeton University, Princeton, New Jersey 08544

P. Toulemonde, S. Le Floch, P. Bordet, and J. J. Capponi

Laboratoire de Cristallographie, CNRS, Avenue des Martyrs 25, F-38042 Grenoble Cedex 9, France

R. Gatt

Department of Physics (M/C 273), 2168 SES, University of Illinois, Chicago, Illinois 60612

W. Tröger, B. Ctortecka, and T. Butz

Fakultät für Physik und Geowissenschaften, Universität Leipzig, Linnéstraße 5, D-04103 Leipzig, Germany

\section{H. Haas}

Bereich Festkörperphysik, Hahn-Meitner-Institut Berlin GmbH, D-14109 Berlin, Germany

J. G. Marques and J. C. Soares

Instituto Tecnológico e Nuclear, Estrada Nacional 10, P-2685 Sacavém, Portugal and CFNUL, Avenida Professor Gama Pinto 2, P-1699 Lisboa Codex, Portugal

(Received 27 October 1999)

\begin{abstract}
Electric-field gradients on mercury sites of the $\mathrm{HgBa}_{2} \mathrm{CuO}_{4+\delta}$ high- $T_{c}$ superconductor were measured with the perturbed angular correlation technique and interpreted with ab initio calculations. Under oxygen annealing, an asymmetric electric-field gradient has been assigned to the presence of single oxygen atoms, $\mathrm{O}_{\delta}$, which are located in the $\mathrm{Hg}$ planes. These experiments provide an atomic scale tool for studying charge-density variations occuring in the neighborhood of the $\mathrm{Hg}$ atoms, which can be induced, particularly, by pointlike defects.
\end{abstract}

\section{INTRODUCTION}

Since the compounds of the family $\mathrm{HgBa}_{2} \mathrm{Ca}_{(n-1)} \mathrm{Cu}_{n} \mathrm{O}_{(2 n+2+\delta)}$ have been synthesized they have been revealed as one of the most interesting systems among high- $T_{c}$ superconductors. ${ }^{1}$ These compounds crystallize in a tetragonal lattice (space group $P 4 / \mathrm{mmm}$ ) with lattice parameters $a \approx a_{\text {perovskite }} \approx 3.88 \AA$ and $c \approx 9.5+3.2(n$ -1) $\AA$ (Fig. 1). While the bond between $\mathrm{Hg}$ and the apical oxygen $\mathrm{O}(2)$ seems to be quite strong and incompressible, the $\mathrm{Cu}-\mathrm{O}(2)$ bond length is pressure dependent. At $30 \mathrm{GPa}$ a critical temperature $\left(T_{c}\right)$ as high as $164 \mathrm{~K}$ can be achieved in the Hg-1223 $(n=2)$ compound. ${ }^{2}$ Such facts created the expectation that precise measurements of the structural properties would provide a better understanding of the chargetransfer mechanisms that generate carriers in the $\mathrm{CuO}_{2}$ superconducting planes. However, it was soon realized that the $\mathrm{Hg}$ planes are particularly disordered. In fact, there are $\mathrm{Hg}$ vacancies, impurities replacing $\mathrm{Hg}$ and the nonstoichiometric oxygen, $\mathrm{O}_{\delta}$, that is considered to be the dopant that regulates $T_{c} .{ }^{3}$ Meanwhile, structural distortions of the po- laronic type and of charge inhomogeneity in the $\mathrm{CuO}_{2}$ layers have become the main issue of research in the field of superconductivity. Neutron-diffraction studies performed on powder samples ${ }^{4}$ as well as more local methods such as extended $x$-ray-diffraction fine structure $^{5}$ pointed to the existence of local distortions in these compounds. So far it is not clear if

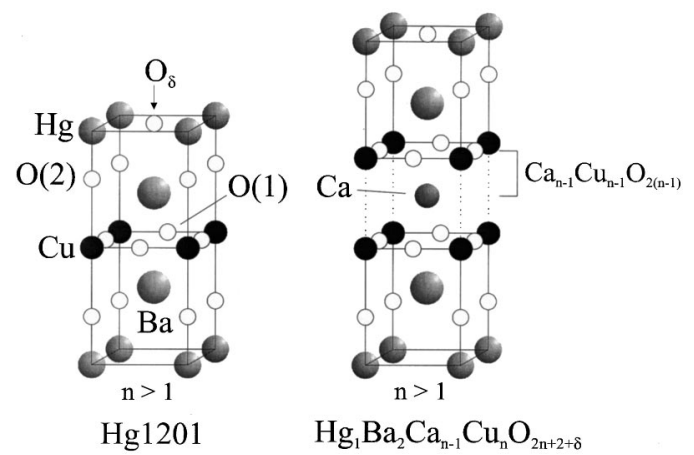

FIG. 1. Schematic view of the $\mathrm{Hg} 1201$ (left) and $\mathrm{HgBa}_{2} \mathrm{Ca}_{n-1} \mathrm{Cu}_{n} \mathrm{O}_{2 n+2+\delta}$ (right) crystalline structures. 


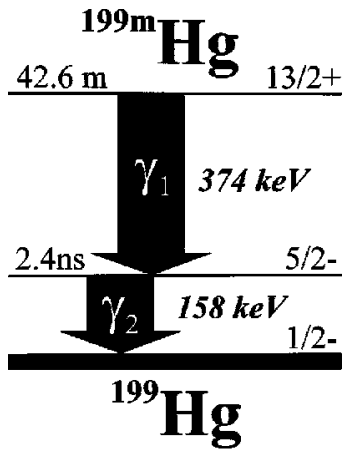

FIG. 2. $\gamma$ - $\gamma$ cascade in the decay of the isomeric state of ${ }^{199 \mathrm{~m}} \mathrm{Hg}$ $\left(T_{1 / 2}=42.6 \mathrm{~min}\right)$ to the ${ }^{199} \mathrm{Hg}$ ground state.

such effects are linked to the superconductivity mechanism or are simply due to crystal-impurity chemical effects.

Nuclear hyperfine techniques like nuclear magnetic resonance (NMR) and nuclear quadrupole resonance (NQR) are extremely useful in providing atomic scale information on the probe-element interactions with the lattice host. ${ }^{6}$ In particular, the measurement of the electric-field gradient (EFG) at specific probe-element nuclei provides direct information about the local charge distribution. The EFG is highly sensitive to the probe's local environment and can thus provide a fingerprint of the lattice site or of a defect that is located in the probe's neighborhood. Unfortunately, NQR on ${ }^{201} \mathrm{Hg}$, the only stable isotope with spin $>1 / 2$, is not too promising and very few experiments on different materials with ${ }^{201} \mathrm{Hg}$ were reported since 1954 by Dehmelt. ${ }^{7}$ Alternatively, time differential perturbed angular correlation (PAC) of $\gamma$ rays, from the decay of the 42.6 min half-life ${ }^{199 m} \mathrm{Hg}$ isomeric state (Fig. 2), is a very well-suited method for this purpose. PAC measures the EFG that is generated by the deviation from cubic symmetry of the charge distribution around the probe nuclei. ${ }^{8}$ In the case of ${ }^{199 m} \mathrm{Hg}$, the interaction of the EFG with the quadrupole moment $Q=0.674 \pm 0.077 \mathrm{~b}$ of the $5 / 2^{-} 158 \mathrm{keV}$ intermediate state of the cascade leads to this level energy splitting. ${ }^{9}$ That induces a time-dependent modulation $G(t)$ on the intensity of the angular correlation between the first gamma ray $\left(\gamma_{1}\right)$, which populates the 158 $\mathrm{keV}$ intermediate state, and the second gamma-ray $\left(\gamma_{2}\right)$ emitted when the intermediate state decays to the ${ }^{199} \mathrm{Hg}$ ground state. Experimentally, $G(t)$ will be observed as a modulation that is superimposed on the half-life decay curve of the $158 \mathrm{keV}$ state.

In the present work, the PAC technique was applied for the first time to investigate the EFG's on $\mathrm{Hg}$ sites of the
Hg1201 $(n=1)$ compound as a function of the oxygen doping concentration.

\section{EXPERIMENTAL}

Hg1201 powder samples were produced by solid-state reaction under high pressure $(18 \mathrm{kbar})$ and high temperature $(1193 \mathrm{~K})$ of a mixture of a precursor with the nominal composition $\mathrm{Ba}_{2} \mathrm{CuO}_{y}$ and the stoichiometric amount of $\mathrm{HgO}$ (in Loureiro $\left.^{10,1(\mathrm{a})}\right)$. The mixture was placed inside a gold capsule for the high pressure-high temperature synthesis. In order to reduce the $\mathrm{O}_{\delta}$ concentration the synthesized material was annealed at $523 \mathrm{~K}$ under 1 bar Ar flow during $24 \mathrm{~h}$. Thereafter, to obtain homogeneous samples the annealed powder was thoroughly mixed in an agate mortar and packed in self-sustained pellet disks, each one with approximately 5 $\mathrm{mm}$ diameter and $0.5 \mathrm{~mm}$ thickness. To avoid contamination or degradation the pellets were stored and manipulated in dry Ar atmosphere. Small samples with approximately $0.5 \mathrm{~mm}^{3}$ were cut out of the disk to be used in the PAC experiments.

The ${ }^{199 m} \mathrm{Hg}$ isotope was produced at the ISOLDE/CERN (Ref. 12) on-line mass separator and implanted into the samples with $60 \mathrm{keV}$ energy under vacuum at room temperature (RT). The ${ }^{199 m} \mathrm{Hg}$ projected range is $R_{P}=17 \mathrm{~nm}$ with the straggling of $\sigma=7 \mathrm{~nm} .{ }^{13}$ In order to produce a homogenous implanted zone and to minimize the implantation damage the ${ }^{199 m} \mathrm{Hg}$ beam was swept to achieve a relatively low dose of $5 \times 10^{11} \mathrm{at} / \mathrm{cm}^{2}$. Under these conditions, $15 \mathrm{~min}$ annealing at $473 \mathrm{~K}$ suffices to eliminate the implantation damage in the ${ }^{199} \mathrm{Hg}$ neighborhood. The experimental setup was equipped with a high-precision temperature controlled furnace where the annealing can be performed during the measurement under gas flow. ${ }^{14}$ For controlling the sample quality and the oxygen concentration, x-ray-diffraction measurements of the lattice parameters were done before and after the PAC experiments on each sample (Table I).

The PAC experiments were performed with a highly efficient 6-detector TDPAC-camera equipped with conical shaped $\mathrm{BaF}_{2}$-scintillators, which has 650 ps (full width at half maximum) time resolution for the ${ }^{199 m} \mathrm{Hg}$ cascade. ${ }^{15} \mathrm{In}$ this setup $6 \gamma_{1}-\gamma_{2}$ coincidence time spectra from detector pairs at $\theta=180^{\circ}$ and 24 coincidence time spectra from detector pairs at $\theta=90^{\circ}$ are simultaneously recorded. With the coincidence countrates, $N_{j}\left(180^{\circ}, t\right), j=1-6$, and $N_{i}\left(90^{\circ}, t\right)$, $i=1-24$, for each detector pair, the experimental ratio $R(t)$ is formed [Eq. (1)]:

TABLE I. $a$ and $c$ lattice parameters measured by x-ray diffraction on the samples before and after the ${ }^{199 m} \mathrm{Hg}$ implantation and the PAC experiments. $\delta$ was estimated from the lattice parameter $a$ as presented in Fig. 5 and explained in the text.

\begin{tabular}{lcccc}
\hline \hline Experiment: $T_{A}, t_{A}$, gas $^{\mathrm{a}}$ & \multicolumn{1}{c}{$a(\AA)$} & $c(\AA)$ & $T_{C}(\mathrm{~K})$ & $\delta(\%)$ \\
\hline As-prepared & $3.8785 \pm 0.0004$ & $9.4998 \pm 0.0013$ & 88 & 10.0 \\
First exp.: 490 K, 220 min, Ar & $3.8801 \pm 0.0005$ & $9.502 \pm 0.002$ & Not measured & 7.9 \\
Third exp.: 490 K, $180 \mathrm{~min}, \mathrm{Ar}$ & $3.8820 \pm 0.0007$ & $9.506 \pm 0.002$ & & 9.1 \\
Second exp.: 490 K, 200 min, $\mathrm{O}_{2}$ & $3.8768 \pm 0.0002$ & $9.4981 \pm 0.0007$ & 10.7 \\
Fourth exp.: 490 K, 240 min, $\mathrm{O}_{2}$ & $3.8758 \pm 0.0009$ & $9.495 \pm 0.003$ & 11.1 \\
\hline \hline
\end{tabular}

${ }^{\mathrm{a}} T_{A}=$ annealing temperature, $t_{A}=$ annealing time. 


$$
R(t)=2 \frac{\sqrt[6]{\Pi_{j}^{6} N_{j}\left(180^{\circ}, t\right)}-\sqrt[24]{\Pi_{i}^{24} N_{i}\left(90^{\circ}, t\right)}}{\sqrt[6]{\Pi_{j}^{6} N_{j}\left(180^{\circ}, t\right)}+2 \sqrt[24]{\Pi_{i}^{24} N_{i}\left(90^{\circ}, t\right)}} .
$$

This ratio eliminates the half-life exponential component, revealing the perturbation function that contains all the relevant information. For each angle $\theta$, the angular correlation functions $W(\theta, t)$ are calculated numerically by taking into account the full Hamiltonian for the nuclear quadrupole hyperfine interaction. ${ }^{8,16}$ Equation (2) defines the theoretical function $R_{\mathrm{fit}}(t)$, whose parameters are fitted to the experimental $R(t)$ function:

$$
R_{\mathrm{fit}}(t)=2 \frac{W\left(180^{\circ}, t\right)-W\left(90^{\circ}, t\right)}{W\left(180^{\circ}, t\right)+2 W\left(90^{\circ}, t\right)} .
$$

For a $\gamma-\gamma$ cascade with the intermediate level of spin $I$ $=5 / 2$, three frequencies are observable per EFG. From these frequencies the coupling constant of the interaction $\nu_{Q}$ $=e Q V_{z z} / h$ and the asymmetry parameter $\eta=\left(V_{x x}\right.$ $\left.-V_{y y}\right) / V_{z z}$ are extracted. $V_{z z}$ is the principal component of the EFG tensor that is produced by the charge distribution outside the probe nucleus. $V_{y y}$ and $V_{x x}$ are the components of the tensor along the $y$ and $x$ axes, which are chosen according to $\left|V_{z z}\right|>\left|V_{y y}\right|>\left|V_{x x}\right|$. In the case of an interaction with randomly distributed defects a distribution of frequencies is observed, which broadens the frequency spectrum and thus attenuates the $R(t)$ function. In this work only weak distributions are found, which were assumed to be Lorentzian-like. These are characterized by the average value $\nu_{Q}$ and standard deviation $\sigma_{Q}$, which depend on the density and variety of the lattice defects. ${ }^{17}$

\section{RESULTS}

\section{A. Experiments}

Figure 3 shows the experimental $R(t)$ functions (left) and the corresponding Fourier transforms (FT's) (right) of four experiments that were performed on the described samples. In order to enhance the quality of the fits, which are represented by continuous lines in the $R(t)$ spectra, the corresponding Fourier transforms of the theoretical functions are included as thicker lines on the FT plots. In order to improve statistics, the summed data taken under the same experimental conditions are also included in Fig. 3. Table II shows the fit parameters. Italic characters are subsequently adopted when referring to fit parameters of added spectra.

When annealing at $489 \mathrm{~K}$ under dry Ar flow, the spectra of the experiments 1 and 3 show a frequency triplet that reveals one axially symmetric $\mathrm{EFG}_{1}$, characterized by $\nu_{Q 1}$ $=1448 \pm 5 \mathrm{MHz}$ and $n_{1}=0$. This clearly corresponds to ${ }^{199 m} \mathrm{Hg}$ nuclei placed on regular sites of the Hg1201 lattice with local tetragonal symmetry. A small attenuation of the $R(t)$ spectra is still observable that is characterized by $\sigma_{Q 1}$ $=35 \pm 3 \mathrm{MHz}$. Most likely the long-range disorder of the $\mathrm{Hg}$ planes produces this small EFG distribution.

When annealing at $489 \mathrm{~K}$ under oxygen flow, the spectra of experiments 2 and 4 exhibit three frequency triplets. Two of them correspond to slightly different EFG's. A fraction $f_{1}=66 \pm 5 \%$ of $\mathrm{Hg}$ nuclei interact with the same axially symmetric $\mathrm{EFG}_{1}$ as above, while a fraction $f_{2}=23 \pm 4 \%$ of



FIG. 3. PAC time spectra $R(t)$ (left) and the corresponding Fourier transforms of the $R(t)$ functions (right). In the $R(t)$ and the Fourier spectra the thicker lines represent the fit function and the Fourier transform of the fit function, respectively.

${ }^{199} \mathrm{Hg}$ nuclei interact with a nonaxially symmetric $\mathrm{EFG}_{2}$, characterized by $\nu_{Q 2}=1229 \pm 14 \mathrm{MHz}$ and $\eta_{2}=0.21 \pm 0.04$. Thus a large fraction of $\mathrm{Hg}$ atoms are on lattice sites with lower than tetragonal symmetry. A third $\mathrm{EFG}_{3}$ was observed which is characterized by $f_{3}=11 \pm 2 \%$ and $\nu_{Q 3}=673$ $\pm 21 \mathrm{MHz}$. In this case the $\eta_{3}$ parameter was set equal to zero, since the small fraction and the overlap of other frequencies does not allow us to determine it unambiguously. Due to the small attenuation of the $R(t)$ spectra $\sigma_{Q 2}=\sigma_{Q 3}$ $=0$ was further assumed for the sake of simplicity.

\section{B. EFG calculations}

In order to interpret the experimental data, EFG calculations were performed with the $a b$ initio full-potentiallinearized-augmented-plane-wave (FLAPW) electronic 


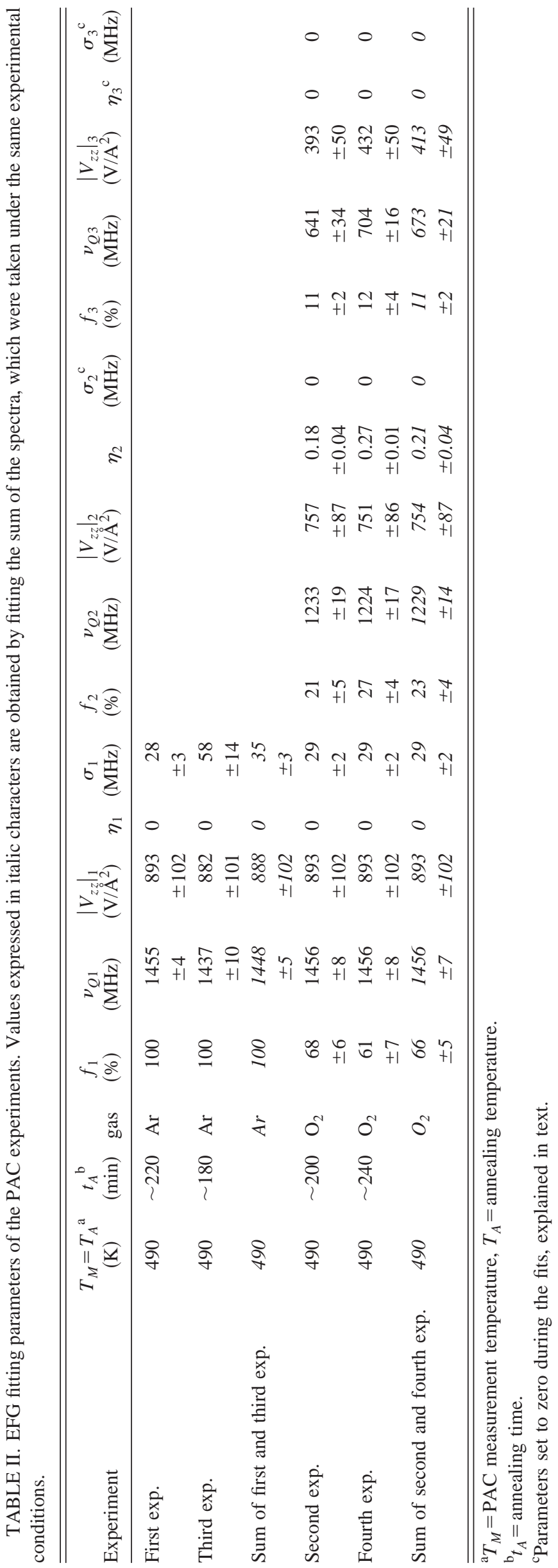

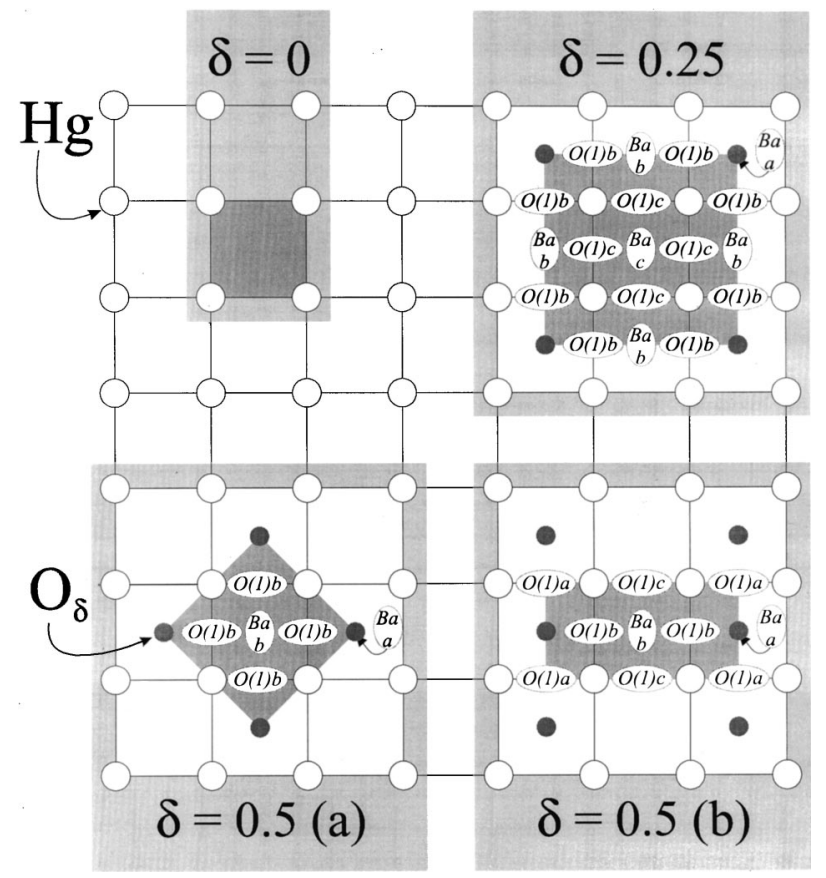

FIG. 4. Representation of the $\mathrm{Hg}$ planes of $\mathrm{Hg} 1201$ as viewed along the $c$ axis. Shaded regions represent the undoped $(\delta=0)$, overdoped ( $\delta=0.25$ ), and saturated $C f(a)$ and $C f(b)(\delta=0.5)$ supercells, which were used for the EFG calculations. Ellipses which enclose italic characters show the position of rows of $\mathrm{Ba} a, \mathrm{Ba} b$, $\mathrm{Bac}$, and $\mathrm{O}(1) a, \mathrm{O}(1) b, \mathrm{O}(1) c$ atoms, which are located out of the $\overline{\mathrm{Hg}}$ planes, as it is explained in the text.

structure method using the local-density approximation. ${ }^{18}$ This method allows, in particular, the computation of hyperfine parameters in crystalline solids by only requiring the lattice constants as input parameters, which in this work were taken from neutron diffraction experiments [Table I in Ref. 11(b)]. To account for the $\mathrm{O}_{\delta}$ doping, several supercells were considered with composition $\mathrm{Hg}_{m} \mathrm{Ba}_{2 m} \mathrm{Cu}_{m} \mathrm{O}_{4 m+n}$. They are denoted as undoped for $\delta=0(m=1, n=0)$, overdoped for $\delta=0.25(m=4, n=1)$ and saturated for $\delta$ $=0.5(m=2, n=1)$. Figure 4 shows a schematic view of the supercells projected along the [001] axis onto the $\mathrm{Hg}$ planes. Table III presents the EFG parameters obtained for the undoped case, for each lattice element. They are in good agreement with previous calculations by Singh. ${ }^{19}$ It should be stressed that the present calculations were performed by setting the internal structure parameter of the apical $\mathrm{O}(2)$ oxygen to $z_{\mathrm{O}(2)}=0.2061$, as obtained from neutron diffraction data. By slightly increasing/decreasing $z_{\mathrm{O}(2)}$ a strong variation of the EFG at the $\mathrm{Hg}$ site was found, e.g., $\left|\partial V_{z Z} / \partial z_{O(2)}\right|_{H g} \approx 2 \times 10^{4} \mathrm{~V} / \AA^{2}$ (see Table III). As expected, the EFG at the $\mathrm{O}(2)$ site has also strongly changed. More surprising is the big influence of small $z_{\mathrm{O}(2)}$ variations on the EFG at the $\mathrm{Ba}$ site, while for $\mathrm{Cu}$ and $\mathrm{O}(1)$ the results are virtually unchanged. Additional information can be obtained from the calculated forces acting on $\mathrm{O}(2)$. When allowing the apical oxygen to relax, while keeping the lattice parameters unchanged elsewhere, one would obtain a theoretical value for $z_{\mathrm{O}(2)}=0.2086$, somewhat different from the experimental data.

In order to calculate the effect of the oxygen doping in the $\mathrm{Hg}$ planes, two saturated $\delta=0.5$ supercells (a) and (b) were constructed (Fig. 4). These correspond to an unrealistic high 
TABLE III. EFG parameters obtained from FLAPW firstprinciples calculations for the undoped $(\delta=0)$ cell. For those positions with a calculated value of $\eta=0$, the principal system of axis (psa) of the EFG has the $z$ axis parallel to [001] and the $x$ and $y$ axes parallel to the [100] and [010] directions. For the $\mathrm{O}(1)$ position the $z$ axis of the psa of the EFG is parallel to [100], along the $\mathrm{Cu}-\mathrm{O}(1)$ bond, the $x$ axis is parallel to [001] and the $y$ axis is parallel to [010]. $a=3.88881 \AA, c=9.5398 \AA, z_{\mathrm{Ba}}=0.302$.

\begin{tabular}{|c|c|c|c|c|c|c|c|}
\hline \multirow[b]{3}{*}{ Element } & \multirow[b]{3}{*}{$V_{i i}$} & \multicolumn{6}{|c|}{$z_{\mathrm{O}(2)}$} \\
\hline & & \multicolumn{2}{|c|}{$\begin{array}{c}\text { Standard } \\
0.2061\end{array}$} & \multicolumn{2}{|c|}{$\begin{array}{c}\text { Compressed } \\
0.2011\end{array}$} & \multicolumn{2}{|c|}{$\begin{array}{c}\text { Expanded } \\
0.2161\end{array}$} \\
\hline & & $\left(\mathrm{V} / \AA^{2}\right)$ & $(\eta)$ & $\left(\mathrm{V} / \AA^{2}\right)$ & $(\eta)$ & $\left(\mathrm{V} / \AA^{2}\right)$ & $(\eta)$ \\
\hline $\mathrm{Hg}$ & $V_{z z}$ & -818.8 & 0 & -929.4 & 0 & -653.4 & 0 \\
\hline $\mathrm{Ba}$ & $V_{z z}$ & 60.4 & 0 & 48.4 & 0 & 85.6 & 0 \\
\hline $\mathrm{Cu}$ & $\begin{array}{l}V_{z z} \\
V_{x x}\end{array}$ & $\begin{array}{l}-28.4 \\
-43.3\end{array}$ & 0 & $\begin{array}{l}-28.2 \\
-42.7\end{array}$ & 0 & $\begin{array}{l}-27 \\
-45.6\end{array}$ & 0 \\
\hline $\mathrm{O}(1)$ & $\begin{array}{l}V_{y y} \\
V_{z z}\end{array}$ & $\begin{array}{r}-83.9 \\
127.2\end{array}$ & 0.318 & $\begin{array}{r}-84.6 \\
127.3\end{array}$ & 0.329 & $\begin{array}{r}-83.2 \\
128.8\end{array}$ & 0.292 \\
\hline $\mathrm{O}(2)$ & $V_{z z}$ & 141 & 0 & 128.8 & 0 & 163 & 0 \\
\hline
\end{tabular}

degree of doping and describe two different configurations of two $\mathrm{O}_{\delta}$ atoms located around every $\mathrm{Hg}$ in relative positions $\left(-1 / 2,-1 / 2, z_{\mathrm{Hg}}\right)\left(1 / 2,1 / 2, z_{\mathrm{Hg}}\right)[C f(a)]$ and $\left(1 / 2,-1 / 2, z_{\mathrm{Hg}}\right)$ $\left(1 / 2,1 / 2, z_{\mathrm{Hg}}\right)[C f(b)]$. In this way the EFG's at the $\mathrm{Hg}$ site produced by two $\mathrm{O}_{\delta}$ neighbors are estimated to be nonaxially symmetric and much weaker then the one found for the undoped case (Tables III and IV). In $C f(a)$ and $C f(b)$ two sites exist for $\mathrm{Ba}$, e.g., $\mathrm{Ba} a$ and $\mathrm{Ba} b$, which are located in and out of $\mathrm{O}_{\delta}$ rows parallel to the [001] axis. $\mathrm{O}(1)$ has only one equivalent site, $\mathrm{O}(1) b$, in $C f(a)$, while it has three inequivalent sites in $\overline{C f(b)}: \mathrm{O}(1) a$ located in between two $\mathrm{O}_{\delta}$ rows, $\mathrm{O}(1) b$ located near only one $\mathrm{O}_{\delta}$ row, and $\mathrm{O}(1) c$ located in sites without $\mathrm{O}_{\delta}$ rows nearby.

Due to Fermi level changes, the EFG at the $\mathrm{Cu}$ site is significantly changed between the undoped and the saturated $C f(a)$ and $C f(b)$ simulations. The EFG is most affected at the $\mathrm{Ba} a$ site where even the sign of $V_{z z}$ has changed, due to the presence of the nearby $\mathrm{O}_{\delta}$ atom (Table IV).

In order to study a more realistic case with smaller oxygen doping concentration, the EFG at the $\mathrm{Hg}$ site with only one $\mathrm{O}_{\delta}$ neighbor in the tetragonal plane has to be calculated. The primary difference between the undoped and the $C f(a)$ saturated supercells is the presence of two $\mathrm{O}_{\delta}$ atoms as neighbors to $\mathrm{Hg}$. Since only moderate changes of the EFG at the $\mathrm{Hg}$ site are calculated, a first guess for the effect of one $\mathrm{O}_{\delta}$ as a neighbor to $\mathrm{Hg}$ may be obtained by interpolating between these cases leading to $V_{z z}^{\text {int }}=751 \mathrm{~V} / \AA^{2}$ and $\eta^{\text {int }}$ $=0.17$.

Further calculations were performed with the overdoped superlattice with $\delta=0.25$ which is close to the maximum $\delta$ $\sim 0.235$ ever found in $\mathrm{Hg} 1201 .{ }^{11}$ Due to the much longer computing time needed for this large supercell, full convergence in the self-consistency cycle was not obtained. The extrapolated EFG parameters obtained from the trend in the calculation are shown in Table IV. There are now three different sites for $\mathrm{Ba}$, e.g., $\mathrm{Ba} a, \mathrm{Ba} b$, and a new $\mathrm{Bac}$ site that corresponds to $\mathrm{Ba}$ atoms which are located out of and farthest away from $\mathrm{O}_{\delta}$ rows. For $\mathrm{O}(1)$ there are only two different possibilities e.g., $\mathrm{O}(1) b$ and $\mathrm{O}(1) c$ (Fig. 4).

The overall consistency of the EFG calculations has been further cross checked for the undoped and overdoped superlattices that approximately reproduce the EFG's at the $\mathrm{Cu}$ site, as measured by $\mathrm{NQR}$ with ${ }^{67} \mathrm{Cu}$ on differently doped Hg1201 materials. ${ }^{20}$

\section{DISCUSSION}

By comparing the experimental $\left|V_{z z}\right|$ values that were obtained when annealing the samples in Ar (Table II) with the calculated one at the $\mathrm{Hg}$ site for the undoped cell (Table III), an excellent agreement is found. Therefore, $\mathrm{EFG}_{1}$ is assigned to $\mathrm{Hg}$ atoms at regular $\mathrm{Hg} 1201$ lattice sites without $\mathrm{O}_{\delta}$ or other defects in the near neighborhood.

The values of $\left|V_{z z}\right|$ and $\eta$ that characterize $\mathrm{EFG}_{2}$, which

TABLE IV. EFG parameters obtained from FLAPW first-particles calculations on the saturated (a) and (b) configurations with $\delta$ $=0.5$ and overdoped with $\delta=0.25$ supercells. For the $\mathrm{Ba} a$ and $\mathrm{Ba} c$ sites the EFG psa is defined as in Table III when $\eta=0$. For the EFG at the $\mathrm{O}(1) a, \mathrm{O}(1) b$, and $\mathrm{O}(1) c$ sites the psa is identical to that of $\mathrm{O}(1)$ in Table III. For the EFG at the $\mathrm{Hg}, \underline{\mathrm{Ba} b}, \mathrm{Cu}, \mathrm{O}(2)$, and $\mathrm{O} \delta$ sites, the psa is now defined with the $z$ axis parallel to [001], the $x$ axis parallel to [110], along the $\mathrm{Hg}-\mathrm{O}_{\delta}$ direction, and the $y$ axis parallel to the $[1-10]$ direction. For the localization of $\underline{\mathrm{Ba} a}, \underline{\mathrm{Ba} b}, \underline{\mathrm{Ba} c}, \underline{\mathrm{O}(1) a}, \underline{\mathrm{O}(1) b}$, and $\underline{\mathrm{O}(1) c}$ in the unit cell see Fig. 4 and text.

\begin{tabular}{|c|c|c|c|c|c|c|c|c|c|c|c|c|}
\hline \multirow[b]{3}{*}{ Element } & \multicolumn{3}{|c|}{$V_{z z}$} & \multicolumn{3}{|c|}{$V_{y y}$} & \multicolumn{3}{|c|}{$V_{x x}$} & \multicolumn{3}{|c|}{$|\eta|$} \\
\hline & \multicolumn{2}{|c|}{ Saturated } & \multirow[t]{2}{*}{ Overdoped } & \multicolumn{2}{|c|}{ Saturated } & \multirow[t]{2}{*}{ Overdoped } & \multicolumn{2}{|c|}{ Saturated } & \multirow[t]{2}{*}{ Overdoped } & \multicolumn{2}{|c|}{ Saturated } & \multirow[t]{2}{*}{ Overdoped } \\
\hline & $C f(a)$ & $C f(b)$ & & $C f(a)$ & $C f(b)$ & & $C f(a)$ & $C f(b)$ & & $C f(a)$ & $C f(b)$ & \\
\hline $\mathrm{Hg}$ & -682.8 & -602.8 & -760 & +457.1 & +305.7 & +468 & +225.7 & +297.1 & +292 & 0.339 & 0.015 & 0.232 \\
\hline $\mathrm{Ba} a$ & -110.6 & -99.6 & -94 & +55.3 & +50.2 & +47 & +55.3 & +49.4 & +47 & 0 & 0.008 & 0.000 \\
\hline$\overline{\mathrm{Ba} b}$ & +46.8 & +34.0 & +46 & -23.4 & -19.2 & -24.5 & -23.4 & -14.8 & -21.5 & 0 & 0.129 & 0.065 \\
\hline$\overline{\mathrm{Ba} c}$ & & & +39 & & & -19.5 & & & -19.5 & & & 0.000 \\
\hline$\overline{\mathrm{Cu}}$ & -52.2 & -58.0 & -13 & +26.9 & +30.6 & +6.5 & +25.3 & +27.4 & +6.5 & 0.031 & 0.055 & 0.000 \\
\hline $\mathrm{O}(1) a$ & & +144.3 & & & -82.1 & & & -62.2 & & & 0.138 & \\
\hline$\overline{\mathrm{O}(1) b}$ & +154.5 & +146.3 & +150 & -84.0 & -84.9 & -76.5 & -70.5 & -61.4 & -73.5 & 0.087 & 0.161 & 0.020 \\
\hline$\overline{\mathrm{O}(1) c}$ & & +144.4 & +148 & & -85.1 & -77.7 & & -59.3 & -70.3 & & 0.179 & 0.050 \\
\hline$\overline{\mathrm{O}(2)}$ & +125.2 & +121.5 & +131 & -67.2 & -65.7 & -67.5 & -58.0 & -55.8 & -63.5 & 0.074 & 0.082 & 0.030 \\
\hline $\mathrm{O}_{\delta}$ & -158.6 & -127.2 & -86 & +79.3 & +80.0 & +43 & +79.3 & +47.2 & +43 & 0 & 0.258 & 0.000 \\
\hline
\end{tabular}


was found when annealing under oxygen flow, compare well with the EFG parameters calculated for $\mathrm{Hg}$ sites with a single near-neighbor $\mathrm{O}_{\delta}$ atoms, as calculated with the overdoped superlattice and by interpolating between the undoped and saturated $C f(a) \mathrm{EFG}$ estimations. Therefore, $\mathrm{EFG}_{2}$ is assigned to $\mathrm{Hg}$ atoms that have one single oxygen atom in their neighborhood in relative coordinates $\left( \pm 1 / 2, \pm 1 / 2, z_{\mathrm{Hg}}\right)$.

At this point it should be stressed that the main contribution for the EFG at the $\mathrm{Hg}$ site comes from the tightly bound apical oxygen, $\mathrm{O}(2)$, that dominates the influence of the weakly $\mathrm{Hg}$-bound $\mathrm{O}_{\delta}$ defect. This is clearly seen in the experimental data, as well in the calculations of the overdoped model, where the presence of a single $\mathrm{O}_{\delta}$ atom mainly changes the asymmetry parameter $\left(\eta_{2} \neq 0\right)$ of the EFG at the Hg site.

For realistic low $\mathrm{O}_{\delta}$ concentrations the PAC fractions should provide information on $\delta$ within the implanted region of the sample. The EFG calculations showed that only the overdoped configuration explains the fraction $f_{2}$ of $\mathrm{Hg}$ nuclei interacting with $\mathrm{EFG}_{2}$. From the atomic point of view $f_{2}$ accounts for the $\mathrm{Hg}$ atoms that have only one $\mathrm{O}_{\delta}$ atom in their neighborhood, which are located at the center of the $\mathrm{Hg}$ square. Since four $\mathrm{Hg}$ atoms surround each $\mathrm{O}_{\delta}$, the corresponding abundance is given by $\delta_{\mathrm{PAC}}=f_{2} / 4 \approx 0.06$.

It should be noted that when annealing under oxygen flow a fraction of ${ }^{199 m} \mathrm{Hg}$ atoms interact with an additional and weaker $\mathrm{EFG}_{3}$. Once $f_{3}$ is small, the full characterization and assignment of $\mathrm{EFG}_{3}$ to a specific $\mathrm{Hg}$-defect configuration cannot be achieved with the present set of data. However, the fact that $\left|V_{z z_{3}}\right|=413 \pm 49 \mathrm{~V} / \AA^{2}$ has a much lower value than expected for configurations of $\mathrm{Hg}$ atoms with two $\mathrm{O}_{\delta}$ nearneighbors, as predicted by the saturated model, hints that $\mathrm{EFG}_{3}$ cannot be due to simple $\mathrm{O}_{\delta}$ defects. Hence, more oxygen-related defects should exist near the $\mathrm{Hg}$ planes in unknown configurations and with unknown oxygen content.

Once the PAC experiments were finished, x-raydiffraction analyses were done on the same samples. These revealed that $a$ and $c$ have changed as expected, i.e., increasing on the samples which have been annealed under Ar and decreasing on the samples which have been annealed under oxygen flow, relative to the lattice parameters of the asprepared material (Table I).

Looking forward to estimate $\delta$ on an independent way from the PAC experiments, Fig. 5 shows the trend of $\delta$ as a function of $a$. The continuous line is a parabolic fit to a set of $\delta$ values which were experimentally measured by several authors and compiled by Loureiro. ${ }^{11}$ Based on this trend and on the x-ray-diffraction data, $\delta$ was estimated in the present samples to be $\delta \approx 0.08$ and $\delta \approx 0.11$ after annealing under $\mathrm{Ar}$ and $\mathrm{O}_{2}$, respectively.

It should be noted that from the PAC experiments, which were performed during annealing under Ar flow, no fraction of $\mathrm{Hg}$ atoms interacting with $\mathrm{O}_{\delta}$ was found $\left(f_{1}\right.$ $\left.=100 \% \Rightarrow \delta_{\mathrm{PAC}}=0\right)$. Only when annealing under oxygen flow was a value for $\delta_{\mathrm{PAC}}=0.06$ found as obtained from the fraction of $\mathrm{Hg}$ atoms that interact with single $\mathrm{O}_{\delta}$ atoms. In both cases $\delta_{\mathrm{PAC}}$ is much smaller than $\delta$ estimated from the lattice parameters.

Besides the uncertainty that characterizes the systematic trend of Fig. 5, different reasons can explain this difference.

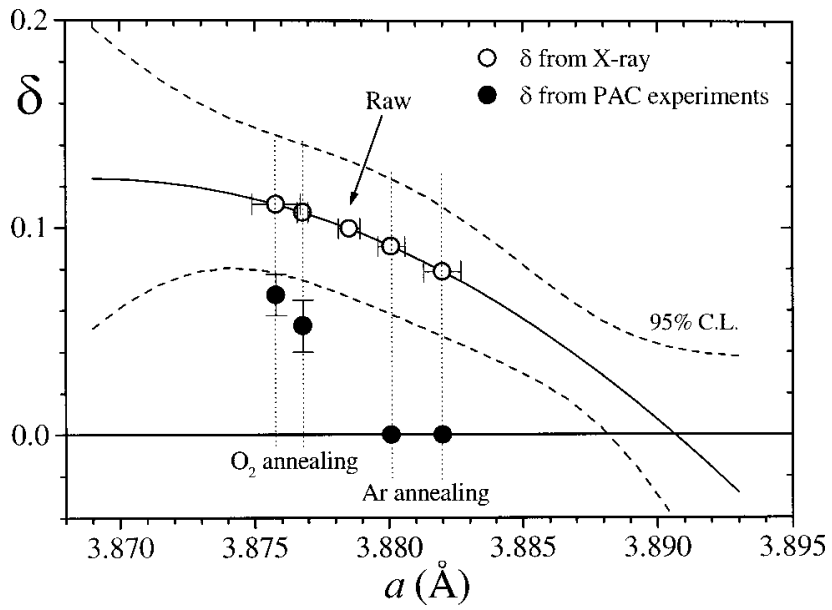

FIG. 5. Trend of $\delta$ as a function of the lattice parameter $a$. The continuous line is a parabolic fit to the experimental data collected in Ref. 11. The dotted lines confine the region of the fit within $95 \%$ confidence level. For the sake of clearness the experimental points where the fit was done are not included in the figure.

The first one is the fact that the shallow implantation depth of the ${ }^{199 m} \mathrm{Hg}$ nuclei is very close to the surface. Consequently, during annealing treatments the ${ }^{199 m} \mathrm{Hg}$ probe nuclei are more exposed to oxygen release than the bulk of the samples that is probed by x-ray analysis, resulting in nonhomogeneous $\mathrm{O}_{\delta}$ concentration. The second reason has to do with the fact that $\delta_{\mathrm{PAC}}$ only accounts for those oxygen atoms that exist as single $\mathrm{O}_{\delta}$ atoms at the center of the $\mathrm{Hg}$ planes without defects nearby. As it was already discussed, the PAC data show that other defect complexes should exist, but, so far, with unknown oxygen content. When using the traditional nonlocal techniques for measuring the oxygen concentration, on which the systematic trend of Fig. 5 is based, single oxygen atoms are not distinguished from other oxygen-related defects. This can lead, in particular, to an overestimation of the concentration of the simple $\mathrm{O}_{\delta}$ defect.

\section{CONCLUSIONS AND PERSPECTIVES}

The EFG's at the Hg site in Hg1201 have been measured with the PAC nuclear technique. Annealing performed under $\mathrm{Ar}$ and $\mathrm{O}_{2}$ flow revealed that the EFG's at the $\mathrm{Hg}$ site are quite sensitive to the $\mathrm{O}_{\delta}$ doping concentration. After oxygen annealing a value of $\delta_{\mathrm{PAC}}=0.06$ is found, which represents the concentration of $\mathrm{O}_{\delta}$ atoms located at the center of the $\mathrm{Hg}$ squares. PAC has further revealed that more unidentified oxygen-related defects must be present near the $\mathrm{Hg}$ planes. These can lead to a misinterpretation of $\delta$ when measured by the more traditional bulk techniques.

EFG first-principles calculations were performed for the undoped and oxygen doped $\mathrm{Hg} 1201$ lattice structure. These reproduced well the experimental values obtained for the EFG at the $\mathrm{Hg}$ site, as well those which were measured by $\mathrm{Machi}^{20}$ for the $\mathrm{Cu}$ site. The ab initio calculations have confirmed that $\mathrm{Hg}$ is tightly bound to the apical oxygen, whose contributions dominate the EFG at the $\mathrm{Hg}$ site. The calculations have further shown that the EFG at the Ba site is extremely sensitive to the $\mathrm{O}_{\delta}$ presence. This fact suggests that an experimental effort should be envisaged to measure the 
EFG's at the $\mathrm{Ba}$ site, in order to probe the changes of the $\mathrm{Ba}$ local charge density and symmetry as a function of doping and temperature.

The reproducibility of the present experiments and the consistency of the theoretical simulations show that PAC experiments on pure and underdoped $\mathrm{Hg} 1201$ samples can provide microscopic information about the $\mathrm{O}_{\delta}$ doping. $\mathrm{Fu}-$ ture experiments should provide details on the $\mathrm{O}_{\delta}$ diffusion mechanisms as well as on the local behavior of $\mathrm{Hg}$ and its electronic environment as a function of temperature.

\section{ACKNOWLEDGMENTS}

This work was partially funded by FCT, Portugal, through Project Nos. CERN/S/FIS/1048/98, PBICT/C/CTM/1891/95, and grants under the PRAXIS XXI Program (J.P.A.), and ITN (J.G.C.).
*On leave from Instituto Tecnológico e Nuclear, Estrada Nacional 10, P-2685 Sacavém, Portugal.

${ }^{1}$ S. N. Putilin, E. V. Antipov, O. Chmaissem, and M. Marezio, Nature (London) 362, 226 (1993); A. Schilling, M. Cantoni, J. D. Guo, and H. R. Ott, ibid. 363, 56 (1993).

${ }^{2}$ L. Gao, Y. Y. Xue, F. Chen, Q. Xiong, R. L. Meng, D. Ramirez, C. W. Chu, J. H. Eggert, and H. K. Mao, Phys. Rev. B 50, 4260 (1994).

${ }^{3}$ M. H. Julien, P. Carretta, M. Horvatic, C. Berthier, Y. Berthier, P. Ségransan, A. Carrington, and D. Colson, Phys. Rev. Lett. 76, 4238 (1996); M. H. Julien, M. Horvatic, P. Carretta, C. Berthier, Y. Berthier, P. Ségransan, S. M. Loureiro, and J. J. Capponi, Physica C 268, 197 (1996); C. H. Booth, F. Bridges, E. D. Bauer, G. G. Li, J. B. Boyce, T. Claeson, C. W. Chu, and Q. Xiong, Phys. Rev. B 52, R15 745 (1995).

${ }^{4}$ P. Bordet, F. Duc, P. G. Radaelli, A. Lanzara, N. Saini, A. Bianconi, and E. V. Antipov, Physica C 282-287, 1081 (1997); M. Lagues, C. F. Beuran, C. Hatterer, P. Laffez, V. Mairet, C. Partiot, X. M. Xie, X. Z. Xu, C. Devill Cavellin, B. Eustache, and C. Coussot, in Coherence in High Temperature Superconductors, edited by Guy Deutscher and Alex Revcolevschi (World Scientific, Singapore, 1996), pp. 70-98.

${ }^{5}$ A. Lanzara, N. L. Saini, Bianconi, F. Duc, and P. Bordet, Phys. Rev. B 59, 3851 (1999).

${ }^{6}$ M.-H. Julien, M. Horvatic, P. Carretta, C. Berthier, Y. Berthier, P. Ségransan, S. M. Loureiro, and J. J. Capponi, Physica C 268, 197 (1996).

${ }^{7}$ H. G. Dehmelt, H. G. Robinson, and W. Gordy, Phys. Rev. 93, 480 (1954).

${ }^{8}$ Th. Wichert, N. Achziger, H. Metzner, and R. Sielemann, Perturbed Angular Correlation in Hyperfine Interactions of Defects in Semiconductors (Elsevier, Amsterdam, 1992), p. 1; T. Butz, Hyperfine Interact. 52, 189 (1989).

${ }^{9}$ W. Tröger, T. Butz, P. Blaha, and K. Schwarz, Hyperfine Interact. 80, 1109 (1989).
${ }^{10}$ S. M. Loureiro et al., Physica C 243, 1 (1995).

${ }^{11}$ (a) S. M. Loureiro, Ph.D. thesis, Université Joseph Fourier, Grenoble, 1997; (b) S. M. Louneiro, J. J. Capponi, E. V. Antipov, and M. Marezio, On the Synthesis and Structure of $\mathrm{HgBa}_{2} \mathrm{Ca}_{n-1} \mathrm{Cu}_{n} \mathrm{O}_{2 n+2+\delta}$ Superconductors, Studies of High Temperature Superconductors Vol. 25, edited by A. V. Narlikar (Nova Science, New York, 1997), and references therein.

${ }^{12}$ E. Kugler, D. Fiander, B. Jonson, H. Haas, A. Przewloka, H. L. Ravn, D. J. Simon, K. Zimmer, and the ISOLDE Collaboration, Nucl. Instrum. Methods Phys. Res. B 70, 41 (1992).

${ }^{13}$ J. F. Ziegler, J. P. Biersack, and U. Littmark, The Stopping and Range of Ions in Solids (Pergamon, New York, 1985).

${ }^{14}$ W. Tröger and T. Butz, Z. Naturforsch., A: Phys. Sci. 47a, 12 (1992).

${ }^{15}$ T. Butz, S. Saibene, Th. Fraenzke, and M. Weber, Nucl. Instrum. Methods Phys. Res. A 284, 417 (1989).

${ }^{16}$ N. P. Barradas, M. Rots, A. A. Melo, and J. C. Soares, Phys. Rev. B 47, 8763 (1993).

${ }^{17}$ M. Deicher, Hyperfine Interact. 79, 681 (1993).

${ }^{18}$ P. Blaha, P. Dufek, K. Schwarz, and H. Haas, Hyperfine Interact. 97/98, 3 (1996); P. Blaha, K. Schwarz, and J. Luitz, WIEN97, Vienna University of Technology 1997 [improved and updated Unix version of the original copyrighted WIEN code, published by P. Blaha. Kschwarz, P. Sonahtin, and S. B. Trickey, Comput. Phys. Commun. 59, 399 (1990)].

${ }^{19}$ D.-J. Singh, Physica C 212, 228 (1993).

${ }^{20}$ T. Machi, R. Usami, H. Yamauchi, N. Koshizuka, and H. Yasuoka, Physica C 235-240, 1675 (1994); W. Hoffmann, H. Breitzke, C. T. Simmons, K. Lüders, G. Buntkowsky, H. H. Limbach, E. V. Antipov, A. A. Gippius, O. Loebich, H. R. Khan, M. Paranthaman, and J. R. Thompson, Appl. Magn. Reson. 8, 57 (1995); Kunisuke Asayama, Yoshio Kitaoka, Guoqing Zheng, and Kenji Ishida, Prog. Nucl. Magn. Reson. Spectrosc. 28, 221 (1996); A. A. Gipius, E. V. Antipov, W. Hoffmann, and K. Lüders, Physica C 276, 57 (1997). 